Mikrochim. Acta 107, 189-196 (1992)

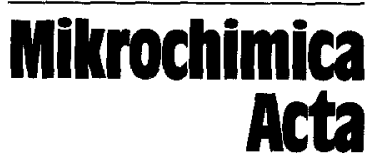

by Springer-Verlag 1992

Printed in Austria

\title{
Distribution and Thickness of the Surface Contaminations on STM Tungsten Tips, Studied by AES/SEM and ARXPS
}

\author{
Wojciech F. Lisowski ${ }^{1,3}$, Albert H. J. van den Berg ${ }^{2}$, Lambertus J. Hanekamp ${ }^{1,2, * \text {, }}$ \\ and Arend van Silfhout ${ }^{1}$ \\ 1 Faculty of Applied Physics and \\ ${ }^{2}$ Centre of Materials Research, University of Twente, P.O. Box 217, 7500 AE Enschede, \\ The Netherlands \\ 3 Permanent address: Institute of Physical Chemistry, Polish Academy of Sciences, \\ ul Kasprzaka 44/52, 01-224, Warszawa, Poland
}

\begin{abstract}
The combination of Auger electron spectroscopy (AES), scanning electron microscopy (SEM) and angle resolved X-ray photoelectron spectroscopy (ARXPS) has been applied to the analysis of the distribution of elements at the surface region of electrochemically etched tungsten tips and the determination of the thickness of a layer with oxygen and carbon contamination. Auger line profiling revealed a homogeneous distribution of oxygen and significant enrichment of carbon on the $\mathrm{W}$ tip between 0 and $1.5 \mu \mathrm{m}$ from the top. The thickness of the contamination layer on various $\mathrm{W}$ materials, electrochemically etched, was found to be $1.35 \pm 0.15 \mathrm{~nm}$ as measured using ARXPS, and was estimated to be about $1-3 \mathrm{~nm}$ as measured by AES.
\end{abstract}

Key words: AES, sputtering, STM, XPS.

One of the important aspects of scanning tunneling microscopy (STM) operation is the reliable formation of tunneling probe tips. Characterization of the surface contaminations on STM tips is helpful for a preliminary selection of good performance tips [1]. It is well known, that the oxides and graphite layers can influence the tunneling current by changing the effective resistance of tip surface layer $[2,3]$. In the light of these results it was interesting to investigate the thickness of these layers.

Transmission electron microscopy (TEM) studies indicated that the most widely applied tungsten tips, prepared by electrochemical etching, were covered by thin $(1-5 \mathrm{~nm}$ thick) polycrystalline tungsten oxides $[3,4]$ and carbon or graphite [5]

\footnotetext{
* To whom correspondence should be addressed
} 
layers. Auger electron spectroscopy (AES) combined with $\mathrm{Ar}^{+}$sputter profiling revealed 1-3 nm thick oxide and carbon layer on W STM tips [1].

The purpose of this paper is the elucidation of the distribution of $\mathrm{C}$ and $\mathrm{O}$ surface contamination both on and in the neighbourhood of the $\mathrm{W}$ tip as well as the determination of the thickness of the contamination layer. In order to realize these objectives, AES combined with $\mathrm{Ar}^{+}$sputter profiling and angle resolved X-ray photoelectron spectroscopy (ARXPS) techniques were utilized. This application, for the first time to STM W tip investigations, of both experimental methods seems to be very useful in obtaining more precise determination of the layer thickness on $\mathrm{W}$ tips.

\section{Experimental}

Electrochemically etched tungsten tips were fabricated by placing some millimeters of $0.8 \mathrm{~mm}$ cold drawn tungsten wire into 1 molar $\mathrm{KOH}$ etching solution and applying a $12 \mathrm{~V}_{\text {eff }} 50 \mathrm{~Hz}$ potential to the tungsten wire (with respect to a gold ring electrode arounding the $\mathrm{W}$ wire and inserted into the solution). The etching process was discontinued when the suspended wire fell off. This wire was cleaned after etching in distilled water and in air directly transported into the measuring system.

Two methods have been used to characterize the composition and the element distribution at the surface region of the $\mathrm{W}$ tip, i.e. Auger line scans and Auger depth profiling using argon ions. The measurements have been performed with a PHI 600 SAM (scanning Auger microscope) system.

The elemental Auger line scans were recorded using the following experimental conditions: a primary beam, perpendicular directed to the axis of the wire, energy $E_{p}=15 \mathrm{kV}$; primary beam current $I_{p}=0.25 \mathrm{nA}$; beam diameter $\Phi \sim 30 \mathrm{~nm} ; 100$ points per line; analysis time per point $\tau=25 \mathrm{~s}$. The resolution of the cylindrical mirror analyzer $(\Delta E / E)$ was set at $1.2 \%$. Under these conditions the detection limit for carbon and oxygen is less than 2 and $0.5 \%$ (atomic concentration) respectively [6]. The line scans have been corrected for topographic effects, by taking the normalised Auger intensity $(P-B) / B$, where $P$ and $B$ stand for the peak and the background intensity respectively [7].

The Auger sputter profiles were taken using $E_{p}=10 \mathrm{kV} ; I_{p}=0.1 \mu \mathrm{A} ; \Phi \sim 0.4 \mu \mathrm{m} ;(\Delta E / E)=0.6 \%$. The axes of the wire, electron beam and ion beam were oriented in one plane. The angle between the axis of the wire and the electron beam and the ion beam was respectively $90^{\circ}$ and $160^{\circ}$. The argon ion beam with an ion energy of $3.5 \mathrm{kV}$ and a current density of $0.6 \mu \mathrm{A} / \mathrm{cm}^{2}$ was produced by a differentially pumped ion gun. The calibration of sputter rate was performed on a $\mathrm{Ta}_{2} \mathrm{O}_{5}$ layer of thickness $100 \mathrm{~nm}$.

The ARXPS experiments were carried out on a KRATOS 800 system, controlled by a PDP 11 microcomputer. The spectrometer was calibrated by measuring the $\mathrm{Ag} 3 \mathrm{~d}_{5 / 2}$ peak and the X-ray induced Ag MNN Auger peak on a clean, sputtered silver sample using a Mg anode [8], and its linearity was checked. The spectra were taken and evaluated using DS 800 software [9]. In all simulations, the asymmetrical Gaussian-Lorentzian mixed functions were used for the tungsten peaks and $100 \%$ Gaussians for the oxide and carbide peaks. Such curve synthesis of W X-ray photoelectron spectra has been investigated by Kojima et al. [10] and was confirmed by our previously reported results [1].

\section{Results and Discussion}

\section{AES Measurements}

Figure 1a shows a SEM image of a STM tungsten tip. The Auger line scans in Fig. $1 \mathrm{~b}$ display the topographically corrected elemental Auger intensity along the tip in the SEM image (Fig. 1 a) indicated by the arrows. 

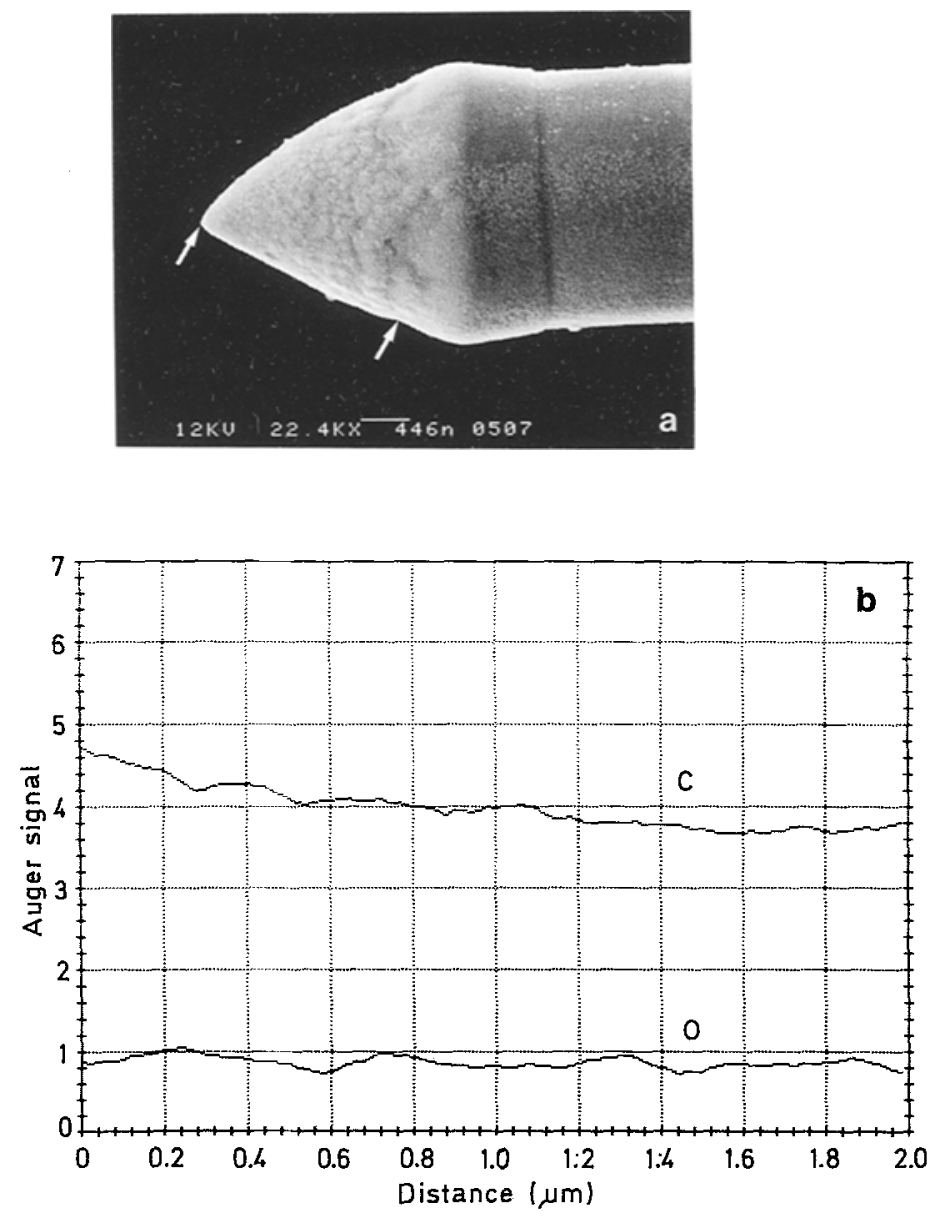

Fig. 1. a Scanning electron microscope image of an electrochemically etched tungsten STM tip analyzed by AES. b Auger elemental linescans, recorded between the two arrows in the SEM image of (a). The Auger signal is plotted in arbitrary units

It can be seen that the oxygen concentration between 0 and $2 \mu \mathrm{m}$ remains constant. The distribution of carbon is not homogeneous. On the top of the W tip a significant enrichment (relative atomic concentration) of carbon has been detected. This can be due to the carbon formation as a result of high local temperature generated during the formation of the tip at the end of an electrochemical etching procedure. Additional Auger line scan measurements revealed a constant concentration of carbon and oxygen between 1.5 and $30 \mu \mathrm{m}$ from the top of the tip.

In order to get a better insight into the distribution and thickness of the carbon and oxygen contamination, we have investigated both the $W$ tip and the large area $W$ samples, i.e. a sheet of the same material and a single crystal of tungsten (purity of $99.999 \%$ ). The electrochemical etching and cleaning procedure were the same as for the $\mathrm{W}$ tip.

Figure 2 shows the measured Auger depth profiles of a tungsten tip (a), a sheet (b) and a single crystal (c). The time required for sputtering of oxygen from all tungsten samples was comparable, which allows to conclude a similar thickness of the oxide layers. The sputter time of carbon was similar for a sheet and single crystal. 


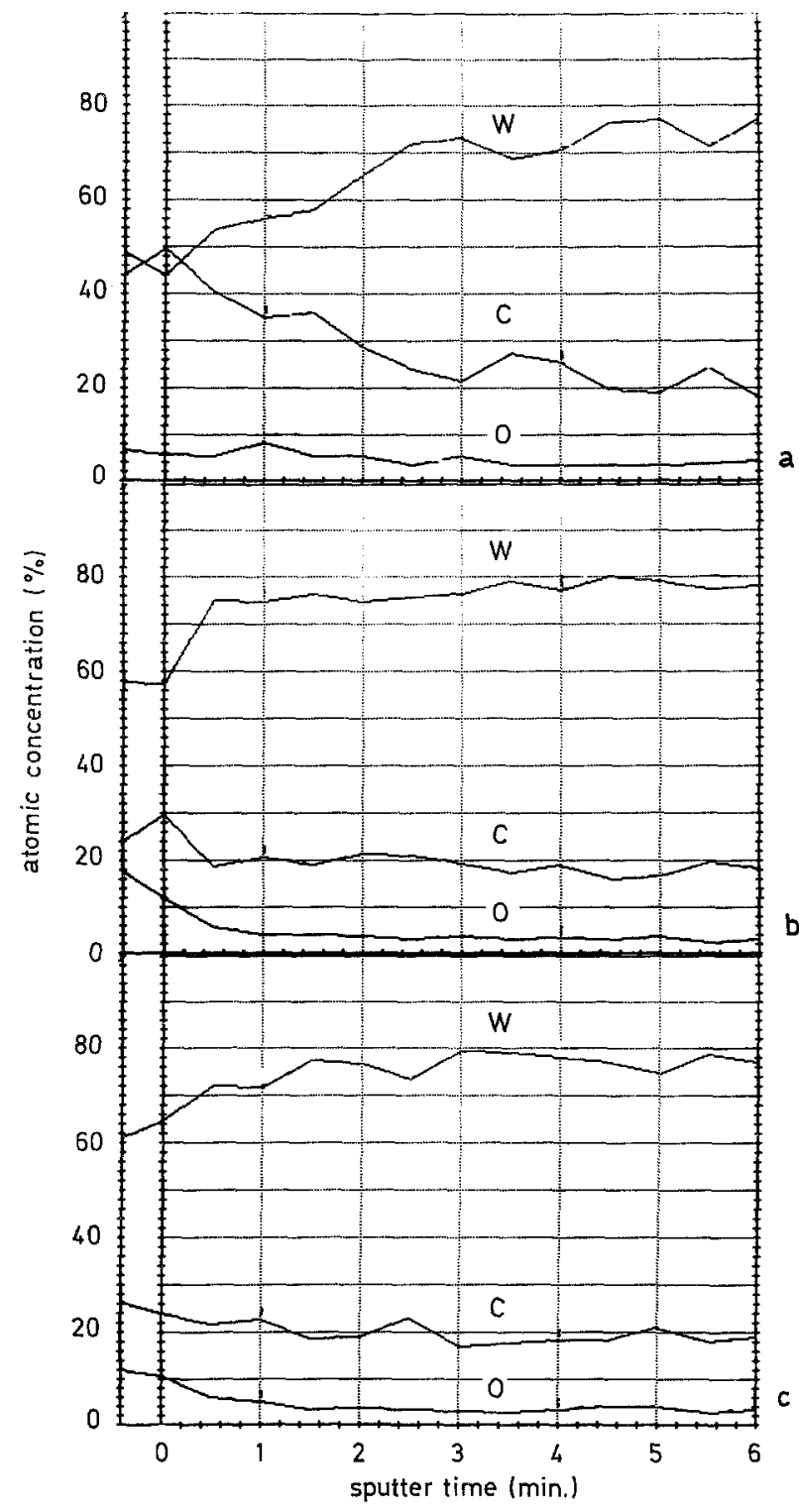

Fig. 2. Auger sputter depth profiles of STM tungsten tip a, W sheet b and W single crystal c. Sputter rate: 0.8 $\mathrm{nm} / \mathrm{min}$

On the tips a longer but variable sputter time was observed. Therefore, for the surface layer thickness estimation, the results of 10 measured $\mathrm{W}$ tips were used.

The calculation of the thickness must be done very carefully. For a rough calculation, a linear relation between sputtering time and removed layer may be assumed. In our system, the sputter rate was found to be $0.8 \mathrm{~nm} / \mathrm{min}$, by calibration performed on a $\mathrm{Ta}_{2} \mathrm{O}_{5}$ layer. Based on the fact that the surface-binding energy for tantalum and tungsten [11], as well as the sputter yield [12] and a depth resolution for $\mathrm{Ar}$ profiling of $\mathrm{Ta}$ and $\mathrm{W}$ [13] are similar, we can use the above value of sputter rate for the estimation of the contamination layer thickness on tungsten. The influence of the ion- and electron-induced effects, accompanying depth-AES profile measurements [14] seems to be comparable for the thin Ta and $\mathrm{W}$ layers, and has been neglected. 
The thickness of the contamination layer was estimated to be 1-2 $\mathrm{nm}$ on the large area $W$ samples and $1-3 \mathrm{~nm}$ on the $W$ tips (the average value for 10 measurements).

In order to verify the above results, the ARXPS measurements were utilized.

\section{ARXPS Measurements}

Non-destructive ARXPS method is used often to study the thickness of the outermost top layer of solid materials [15]. We have applied "absolute" ARXPS measurements described lately by Aarnink et al. [15]. In this method the same XPS peak is measured at varying photoelectron take-off angle and its intensity is normalized to the normal take-off angle.

For the intensity of the XPS peak of tungsten, measured at different electron take-off angles $(\alpha)$ and normalized to its intensity at normal take-off angle $(\alpha=0)$, we may write [15]:

$$
N(\alpha)=\frac{I_{\mathrm{W}, 4 \mathrm{f}}(\alpha)}{I_{\mathrm{W}, 4 \mathrm{f}}(0)}=G(\alpha) \exp \left[\frac{d}{\lambda_{\mathrm{W}, 4 \mathrm{f}, \text { surf }}}\left(1-\frac{1}{\cos \alpha}\right)\right]
$$

where $d$ is a uniform thickness of surface layer and $\lambda_{\mathrm{W}, 4 \mathrm{f} \text {, surf }}$ describes the inelastic mean free path of the photoelectrons in this layer.

The function $G(\alpha)$, depending upon the geometry of the experimental set up, sample and sample holder, has been measured directly on a clean, sputtered silver sample using a single, well separated low BE component of the Ag 3d peak;

$$
G(\alpha)=\frac{I_{\mathrm{Ag}, 3 d}(\alpha)}{I_{\mathrm{Ag}, 3 d}(0)}
$$

The measured function $G(\alpha)$ was close to that obtained on a clean sputtered $\mathrm{Si}$ sample [15].

The thickness of the contamination layer on the sheet and the single crystal of tungsten has been measured. The XPS analysis of the W tip is rather doubtful because a correct take-off angle evaluation on a very small measuring spot is difficult to determine. Nevertheless, the comparable AES results presented here for the various tungsten materials, justify our ARXPS measurements on a large area W samples.

To obtain the intensities of the $W$, we simulated the W4f spectra, taken at $0,10, \ldots 60$ degrees take off angle. In Fig. $3 \mathrm{a}$, two XPS spectra of the W4f peak measured on the single crystal are shown, taken at electron take-off angles $\alpha$ of 0 and 60 degrees respectively. Two Gaussians and one Lorentz-Gaussian doublet were used in the simulation (Fig. $3 \mathrm{~b}$ ). The thinner lines (denoted b, c) represent the $\mathrm{W} 4 \mathrm{f}$ doublets of $\mathrm{WC}$ and $\mathrm{WO}_{3}$ and lines (a) describe pure $\mathrm{W} 4 \mathrm{f}$ doublet of tungsten. The sum of these $\mathrm{W}$ lines is given as a thicker solid line.

The thickness determination has been performed with the W4f low BE component (the line with the strongest intensity), normalized to the intensity at normal take off angle and divided by the measured function $G(\alpha)$.

The result of the application of Eq. (1) for the surface layer thickness determination is presented in Fig. 4. In the coordinate system: 



Fig. 3. a W4f XPS peaks of the single crystal, taken at photoelectron take-off angles of 0 and 60 degrees respectively. b Simulation of the W4f XPS spectrum, taken at 0 degrees take-off angle

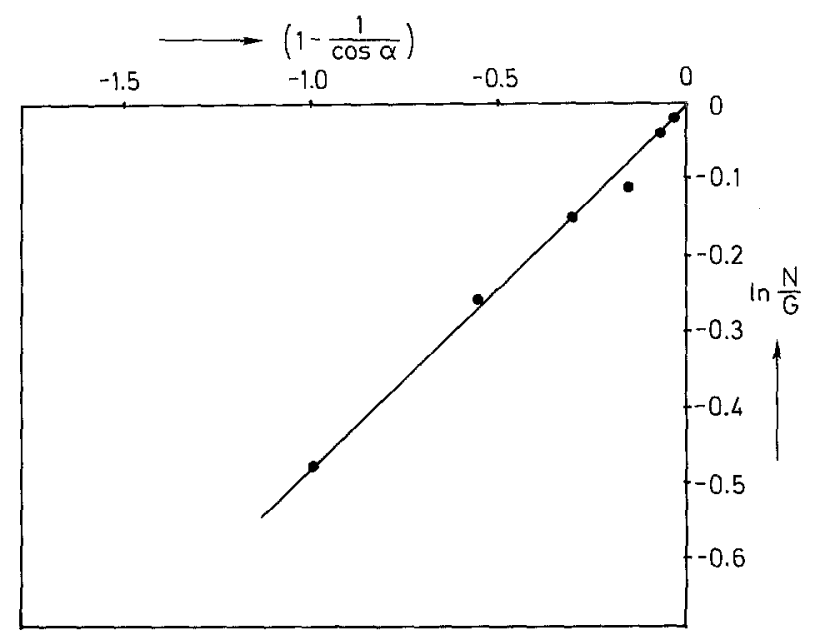

Fig 4. Examination of Eq. (1) for the determination of the thickness of the contamination layer of oxygen and carbon on tungsten using ARXPS measurement. The points represent experimental results, while the solid straight line is the result of the fit 
Table 1. The linear regression parameters of eq. (1), obtained for a $\mathrm{W}$ sheet and a $\mathrm{W}$ single crystal

\begin{tabular}{|l|c|c|}
\hline Material & $\left(\frac{d}{\lambda_{\mathrm{W}, 4 \mathrm{f}, \text { surface }}}\right)$ & Correlation factor \\
\hline W sheet & 0.40 & 0.978 \\
\hline W single crystal & 0.45 & 0.997 \\
\hline
\end{tabular}

$$
\ln \frac{N(\alpha)}{G(\alpha)} \text { vs. }\left(1-\frac{1}{\cos \alpha}\right)
$$

a straight line was obtained. In Table 1 are presented the results of a linear regression analysis of the experimental points obtained for a sheet and a single crystal of tungsten.

Taking a $\lambda_{\mathrm{w}, 4 \mathrm{f} \text {, surf }}$ value from the literature $[16,17]$ we can estimate the thickness $\mathrm{d}$ to be $1.35 \pm 0.15 \mathrm{~nm}$, as an average for two materials. There is good agreement with AES experimental results obtained on the sheet and single crystal of tungsten.

\section{Conclusions}

The following conclusions can be drawn:

- Auger line profiling and AES combined with $\mathrm{Ar}^{+}$sputter profiling techniques appear to be very useful in analysis of both the distribution of the oxygen and carbon contamination on the $\mathrm{W}$ tip and the thickness estimation of this layer.

- The distribution of oxygen contaminations was found to be homogeneous on a W tip.

- The relative higher concentration of carbon was detected between 0 and $1.5 \mathrm{~nm}$ from the W tip top.

- The thickness of the contamination layer was estimated to be $1-2 \mathrm{~nm}$ and 1-3 $\mathrm{nm}$ for the large area $\mathrm{W}$ samples and the $\mathrm{W}$ tips respectively, using AES measurements.

- The thickness of the contamination layer on the various tungsten materials, electrochemically etched, was found to be $1.35 \pm 0.15 \mathrm{~nm}$ using the absolute ARXPS measurements.

Acknowledgements. The authors would like to thank Mr. B. Otten for preparing the SEM images.

\section{References}

[1] W. Lisowski, A. H. J. van den Berg, G. A. M. Kip, L. J. Hanekamp, Fresenius' Z. Anal. Chem. 1992 (in press)

[2] Y. Kuk, P. J. Silverman, Appl. Phys. Lett. 1986, 48, 1597. 
[3] D. K. Biegelsen, F. A. Ponce, J. C. Tramontana, S. M. Koch, Appl. Phys. Lett. 1987, $50,696$.

[4] J. Garnaes, F. Kragh, K. A. Morch, A. R. Thölén, J. Vac. Sci. Technol. 1990, A8, 441.

[5] T. Tiedje, J. Varon, H. Deckman, J. Stokes, J. Vac. Sci. Technol. 1988, A6, 372.

[6] Detectability limits for Auger analysis, PHI Techn. Bull. T8401,1984 May 5, Physical Electronics Industries, Perkin-Elmer.

[7] H. E. Bishop, in: Methods of Surface Analysis (J. M. Walls, ed.), Cambridge University Press, Cambridge, 1989, p. 108.

[8] D. Briggs, M. P. Seah, Practical Surface Analysis by Auger and X-ray Photoelectron Spectroscopy, Wiley, Chichester, 1983, p. 431.

[9] Kratos XSAM 800, Users Guide.

[10] I. Kojima, M. Kurahashi, J. Electron Spectrosc. Relat. Phenom. 1987, 42, 177.

[11] K. A. Gschneider, Solid State Phys. 1964, 16, 275.

[12] M. P. Seah, Thin Solid Film 1981, 81, 279.

[13] H. H. Andersen, Appl. Phys. 1979, 18, 131.

[14] S. Strop, Spectrochim. Acta 1985, 40B, 745.

[15] W. A. M. Aarnink, A. Weishaupt, A. Van Silfhout, Appl. Surf. Sci. 1990, 45, 37 (references therein).

[16] M. P. Seah, W. Dench, Surf. Interf. Anal. 1979, 1, 2.

[17] D. R. Penn, J. Electr. Spectrosc. Relat. Phenom. 1976, 9, 29.

Received June 10, 1991. 